\title{
Acidic pH Triggers Lipid Mixing Mediated by Lassa Virus GP
}

\author{
Uriel Bulow ${ }^{1}$, Ramesh Govindan ${ }^{1,2}$ and James B. Munro 1,2,3,*(D) \\ 1 Department of Molecular Biology and Microbiology, Tufts University School of Medicine, \\ Boston, MA 02111, USA; uriel.bulow@tufts.edu (U.B.); ramesh.govindan@tufts.edu (R.G.) \\ 2 Department of Microbiology and Physiological Systems, University of Massachusetts Medical School, \\ Worcester, MA 01605, USA \\ 3 Department of Biochemistry and Molecular Pharmacology, University of Massachusetts Medical School, \\ Worcester, MA 01605, USA \\ * Correspondence: james.munro@umassmed.edu
}

Received: 12 June 2020; Accepted: 30 June 2020; Published: 2 July 2020

\begin{abstract}
Lassa virus (LASV) is the causative agent of Lassa hemorrhagic fever, a lethal disease endemic to Western Africa. LASV entry is mediated by the viral envelope glycoprotein (GP), a class I membrane fusogen and the sole viral surface antigen. Previous studies have identified components of the LASV entry pathway, including several cellular receptors and the requirement of endosomal acidification for infection. Here, we first demonstrate that incubation at a physiological temperature and $\mathrm{pH}$ consistent with the late endosome is sufficient to render pseudovirions, bearing LASV GP, non-infectious. Antibody binding indicates that this loss of infectivity is due to a conformational change in GP. Finally, we developed a single-particle fluorescence assay to directly visualize individual pseudovirions undergoing LASV GP-mediated lipid mixing with a supported planar bilayer. We report that exposure to endosomal $\mathrm{pH}$ at a physiologic temperature is sufficient to trigger GP-mediated lipid mixing. Furthermore, while a cellular receptor is not necessary to trigger lipid mixing, the presence of lysosomal-associated membrane protein 1 (LAMP1) increases the kinetics of lipid mixing at an endosomal $\mathrm{pH}$. Furthermore, we find that LAMP1 permits robust lipid mixing under less acidic conditions than in its absence. These findings clarify our understanding of LASV GP-mediated fusion and the role of LAMP1 binding.
\end{abstract}

Keywords: Lassa virus; virus entry; membrane fusion; single-particle fusion

\section{Introduction}

Lassa virus (LASV) is an arenavirus endemic to Western Africa. An outbreak of Lassa fever in Nigeria led to 4841 suspected cases and 197 confirmed deaths in the first twenty-one weeks of 2020 [1]. The sequelae of Lassa fever include sensorineural deafness in $25 \%$ of survivors and a greater than $80 \%$ chance of maternal and/or fetal death in pregnant patients in their third trimester [2,3]. Due to the deadly nature of Lassa hemorrhagic fever, LASV is classified as a Category A priority pathogen by the United States Centers for Disease Control and Prevention.

The LASV envelope glycoprotein (GP) coordinates the fusion of the viral envelope with the endosomal membrane of the target cell [4]. GP is synthesized as a precursor glycoprotein complex that is subsequently processed by cellular proteases into subunits GP1 and GP2, as well as a long and stable signal peptide (SSP) [5,6]. During viral entry, GP1 is responsible for binding at least two identified cellular receptors, $\alpha$-dystroglycan $(\alpha \mathrm{DG})$ and lysosomal-associated membrane protein 1 (LAMP1) [7,8]. Studies to date suggest a model in which GP1 first binds $\alpha$-DG on the cell surface, which causes the cell to internalize the virus into an endosome via macropinocytosis [9]. The acidification of the late 
endosome causes GP to undergo an affinity switch from $\alpha$ DG to LAMP1 [10]. Implicated in this affinity switch are histidines $\mathrm{H} 92, \mathrm{H} 93$, and H230, which are located adjacent to the GP1 receptor-binding site and undergo conformational changes in response to protonation [11,12]. A crystallographic structure of GP at a neutral $\mathrm{pH}$ and cryo-electron tomography structures determined under acidic conditions have shown that acidic $\mathrm{pH}$ is sufficient to initiate conformational changes in GP1 [13,14].

The prevailing model of class I fusion holds that GP2 sits in a kinetically trapped, metastable pre-fusion conformation. The transition to the post-fusion conformation is facilitated by a trigger, which lowers the activation energy for exothermic collapse [15]. During fusion triggering, the GP2 subunit undergoes putative conformational rearrangements that involve the adoption of a transient extended intermediate in which the fusion loop is inserted into the target membrane [16]. The subsequent refolding of GP2 brings the target and viral membranes into close approximation, inducing fusion. Like other class I viral fusogens, the formation of the six-helix bundle is thought to drive membrane fusion [17-19].

Previous studies in LASV and lymphocytic choriomeningitis virus (LCMV) have highlighted the importance of acidic $\mathrm{pH}$ in arenavirus entry [14,20-26], with some cell-cell fusion studies suggesting that a $\mathrm{pH}$ as low as 3.5 may be required for LASV GP-mediated fusion to occur $[27,28]$. Of particular note, exposure to a $\mathrm{pH}$ consistent with the late endosome at a physiological temperature was shown to trigger the fusion of LCMV [29]. Here, we show that exposure to low $\mathrm{pH}$ is sufficient at a physiological temperature to trigger conformational changes in LASV GP related to membrane fusion. Furthermore, we show directly by a single-particle fusion assay that exposure to $\mathrm{pH} 5.0$ is sufficient to trigger LASV GP-mediated lipid mixing in the absence of receptor binding. Finally, we demonstrate conclusively that LAMP1 binding is dispensable for LASV GP-mediated lipid mixing. However, its presence in the target membrane increases the kinetics of lipid mixing, facilitating robust lipid mixing at a less acidic $\mathrm{pH}$.

\section{Materials and Methods}

\subsection{Cell Culture}

HEK293T cells were cultured in DMEM (Thermo Fisher, Waltham, MA, USA) supplemented with $10 \%$ cosmic calf serum (GE Healthcare, Chicago, IL, USA), L-glutamine (Thermo Fisher), and penicillin-streptomycin (Thermo Fisher) at $37{ }^{\circ} \mathrm{C}$ and $5 \% \mathrm{CO}_{2}$ and were used to generate pseudovirions as described below. Vero cells were cultured in DMEM supplemented with $10 \%$ fetal bovine serum (FBS; Gemini Bio), L-glutamine, and penicillin-streptomycin at $37{ }^{\circ} \mathrm{C}$ and $5 \%$ $\mathrm{CO}_{2}$ and were used for infectivity assays with pseudovirions bearing GP and HA. DF1 chicken fibroblast cells were cultured in RPMI (Thermo Fisher) supplemented with 10\% FBS, L-glutamine, and penicillin-streptomycin at $37{ }^{\circ} \mathrm{C}$ and $5 \% \mathrm{CO}_{2}$, and were used for infectivity assays with pseudovirions bearing ALV env, as mammalian cells are neither susceptible nor permissive to infection by ALV subtype A.

\subsection{Plasmid DNA}

The pcDNA3 plasmid encoding LASV GP from the Josiah strain was provided by Dr. Melinda Brindley at the University of Georgia [11]. The pVRC8400 plasmid encoding IAV HA (A/Vietnam/2004) was provided by Dr. Peter Kwong at the Vaccine Research Center. HA0 was formed by site-directed mutagenesis as previously described [30]. ALV subtype A Env was provided by Dr. John Coffin at Tufts University. The soluble LAMP1 expression construct was provided by Dr. Juha Huiskonen at the University of Oxford [14].

\subsection{Pseudotype Production}

HEK293T cells were transfected with $10 \mu \mathrm{g}$ of plasmid containing the glycoprotein of interest in a $10 \mathrm{~cm}$ dish at $80 \%$ confluency. PEI MAX was used for transfection at a mass ratio of 4:1 PEI to plasmid 
DNA. Cells were infected with VSV $\Delta$ G-GFP-VSVG (Kerafast) $24 \mathrm{~h}$ post transfection at an MOI of 3 [31]. Supernatant was collected $24 \mathrm{~h}$ post infection and ultra-centrifugated over a $10 \%$ sucrose cushion at $160,000 \times g$ for $90 \mathrm{~min}$. Supernatant was aspirated and pellets were resuspended in phosphate-buffered saline (PBS). The pseudovirus was aliquoted and frozen at $-80{ }^{\circ} \mathrm{C}$ until use. Bald particles were generated following an identical protocol, with the exception of the omission of a glycoprotein.

\subsection{Virus Infectivity Assays}

Infections were performed in Vero cells for studies with LASV GP and IAV HA, and in DF-1 cells for ALV Env. $10 \mu \mathrm{L}$ of concentrated pseudovirus were diluted in $0.1 \mathrm{M}$ phosphate buffer (for $\mathrm{pH} 7.0$ and 6.0) or $0.1 \mathrm{M}$ acetate buffer (for $\mathrm{pH} 5.5$ through 4.0) in temperature-controlled conditions. Virions were then brought back to neutral $\mathrm{pH}$ in $5 \times$ volume of $0.1 \mathrm{M}$ phosphate buffer at $\mathrm{pH} 7.0$ while still at their experimental temperatures, and then immediately serially diluted in room temperature DMEM for infection.

Target cells were infected with the pre-incubated virus and were incubated for one hour at $37^{\circ} \mathrm{C}$ with rocking every $15 \mathrm{~min}$. Fresh DMEM was then added to the cells. At $5 \mathrm{~h}$ post infection, cells were collected by trypsinization and assayed for GFP expression using flow cytometry on a FACSCALIBUR. Statistical significance was calculated using a two-way ANOVA with an alpha of 0.05.

\subsection{LASV GP Antibody Capture ELISA}

Purified VSV pseudotypes were treated with either a phosphate buffer at $\mathrm{pH} 6.0$ or 7.0 , or acetate buffer at $\mathrm{pH} 5.5$ or 5.0 at $37^{\circ} \mathrm{C}$ or on ice for $30 \mathrm{~min}$. The virions were then brought back to a neutral $\mathrm{pH}$, as described above, at their respective temperatures and bound to an ELISA plate. The plate was then blocked with a $3 \%$ BSA solution for $1 \mathrm{~h}$ and washed with PBS three times. The immobilized virions were then probed with non-neutralizing $(26.5 \mathrm{E}, 24.6 \mathrm{C})$ or neutralizing antibodies $(12.1 \mathrm{~F}, 37.2 \mathrm{D}, 37.7 \mathrm{H})$ for $1 \mathrm{~h}$, washed three times with PBS, and probed with an HRP-conjugated rabbit anti-human IgG1 antibody (Abcam, Cambridge, UK) [32]. The plate was developed with SureBlue ${ }^{\mathrm{TM}}$ TMB 1-Component Microwell Peroxidase Substrate (SeraCare, Milford, MA, USA) for twenty minutes and read in a Synergy H1 Biotek plate reader.

\subsection{LAMP1 Purification}

HEK293F cells were transfected with plasmid encoding the full lumenal domain of LAMP1 with a $6 \times$ His tag and grown for 6 days. PEI MAX was used as the transfection reagent as indicated above. Supernatant was collected and circulated over Ni-NTA agarose beads (Thermo Fisher, Waltham, MA, USA) overnight at $4{ }^{\circ} \mathrm{C}$. The beads were then washed with PBS with $50 \mathrm{mM}$ imidazole. LAMP1 was eluted from the beads with PBS with $250 \mathrm{mM}$ imidazole. The protein was then dialyzed into PBS and stored at $-80^{\circ} \mathrm{C}$ in frozen aliquots.

\subsection{Liposome Formation}

Liposomes were formed as described in Floyd et al. [33]. Briefly, 1-oleoyl-2-palmitoyl-sn-glycero-3-phosphocholine (POPC) (Avanti Polar Lipids, Alabaster, AL, USA), 1,2,dioleoyl-sn-glycero-3-phosphocholine (DOPC) (Avanti Polar Lipids, Alabaster, AL, USA), cholesterol, 1,2-dioleoyl-sn-glycero-3-phosphoethanolamine-N-(carboxyfluorescein) (FLPE) (Avanti Polar Lipids, Alabaster, AL, USA), and either 1,2-dioleoyl-sn-glycero-3-[(N-(5-amino-1-carboxypentyl)iminodiacetic acid)succinyl] (Ni-NTA DOGS) (Avanti Polar Lipids, Alabaster, AL, USA) or bovine ganglioside GD1a (Millipore Sigma, Burlington, MA, USA) were mixed at a molar ratio of 4:4:2:0.2:0.1. Chloroform was evaporated under nitrogen gas, and lipids were resuspended in PBS to a working concentration of $10 \mathrm{mg} / \mathrm{mL}$. The lipid mixture underwent seven freeze/thaw cycles in liquid nitrogen followed by extrusion through a $200 \mathrm{~nm}$ pore size polycarbonate membrane filter (Whatman, Maidstone, UK) 21 times. 


\subsection{Labeling Virions}

Virions were labeled by incubating with $2 \mathrm{mM}$ DiD (Thermo Fisher, Waltham, MA, USA) for two hours at room temperature. Unbound DiD was removed by passage over a Zeba desalting column (Thermo Fisher, Waltham, MA, USA) equilibrated in PBS. Labeled virus was used immediately in single-particle lipid mixing assays.

\subsection{Single-Virion Lipid Mixing Assay}

The single-virion lipid mixing assay was adapted from that described by Floyd et al. [33]. Pre-drilled quartz slides and glass coverslips were immersed in distilled water and sonicated for $20 \mathrm{~min}$. They were then moved to $1 \mathrm{M} \mathrm{KOH}$ and the sonication was repeated. The slides were rinsed with distilled water, before being sonicated in acetone, and rinsed again. The coverslips and slides were then allowed to dry overnight. The dry slides were then cleaned in an plasma cleaner (Harrick Plasma, Ithaca, NY, USA) for $2 \mathrm{~min}$. Flow cells were then constructed with the slides and coverslips. Each flow cell was rinsed with PBS, and then $100 \mu \mathrm{L}$ of liposomes were injected into the flow cell by a syringe pump at $80 \mu \mathrm{L} / \mathrm{min}$. The liposomes were allowed to incubate for twenty minutes at room temperature before the channel was again rinsed with $300 \mu \mathrm{L}$ of PBS. Labeled virions were then pumped into the channel at $40 \mu \mathrm{L} / \mathrm{min}$ and allowed to incubate for $20 \mathrm{~min}$ to allow virions to bind to the membrane. The channel was then rinsed with PBS again to remove unbound virus. The slide was then placed on the stage of a prism-based TIRF microscope. Pre-warmed buffer was pumped into the flow cell while acquiring movies at $100 \mathrm{~ms}$ exposures $(10 \mathrm{~Hz})$ for a minimum of $3 \mathrm{~min}$, or until no more dequenching events could be seen. The analysis of the dequenching events was performed in Matlab (MathWorks, Waltham, MA, USA). The interval between 50\% loss of CF fluorescence resulting from acidification and DiD dequenching was extracted for each event and compiled into histograms. The histograms displayed a rise and fall in the frequency of lipid mixing events. This indicates that multiple rate-limiting steps are necessary before the arrival of lipid mixing; a single rate-limiting step would have given rise to an exponentially distributed histogram of the frequency of events. We therefore considered the kinetic scheme

$$
A \rightarrow I_{1} \rightarrow I_{2} \rightarrow \cdots \rightarrow I_{N} \rightarrow B
$$

where $A$ represents the pre-fusion state, $B$ represents the state after lipid mixing, and $I_{1}, I_{2}, \ldots I_{N}$ are the $N$ rate-limiting intermediate states. The probability density function for this scheme, which describes the distribution of time intervals observed for the transit from $A$ to $B$, is the gamma distribution

$$
P(t)=\frac{k^{N} t^{N-1}}{\Gamma(N)} e^{-k t}
$$

where $k$ is the rate constant and $N$ is the number of rate-determining steps. The histograms of the time intervals were fit to the gamma distribution using a least squares algorithm in Matlab. The mean time to dequenching was then determined by calculating $\langle t\rangle=N / k$.

\section{Results}

\subsection{The $p H$ Sensitivity of LASV GP}

We first sought to determine the $\mathrm{pH}$ sensitivity of LASV GP with a viral inactivation assay, a common strategy used to study the $\mathrm{pH}$ dependence of viral fusogen function [34]. We therefore formed GFP-encoding vesicular stomatitis virus (VSV) pseudovirions bearing GP from the Josiah strain of LASV. This pseudotyping strategy has previously been used for mutagenic studies of LASV GP, as well as for LASV vaccine trials $[11,35]$. VSV-GP pseudovirions were first incubated at $21^{\circ} \mathrm{C}$ for five or thirty minutes in buffer at $\mathrm{pH}$ values ranging from 5.0 to 7.0. The virions were returned to a neutral $\mathrm{pH}$ while maintaining the temperature before they were used to infect Vero cells. GFP 
expression was assayed using flow cytometry five hours post infection. Above $\mathrm{pH}$ 5.0, no loss of infectivity was seen after five minutes of incubation at $21{ }^{\circ} \mathrm{C}$ (Figure 1A). Infectivity was reduced by approximately $13 \%$ when the virions were incubated at $\mathrm{pH} 5.0$ for five minutes. A similar loss of infectivity was seen for pseudovirions carrying $\mathrm{H} 5$ hemagglutinin (HA) from influenza, which is known to be triggered by acidic $\mathrm{pH}[34,36]$. When the virions were incubated at $37^{\circ} \mathrm{C}$ and $\mathrm{pH} 5.5$ for five minutes, infectivity was reduced to $45 \%$. Infectivity was further reduced by $90 \%$ when incubated at $\mathrm{pH} 5.0$ (Figure 1A). When the incubation was extended for thirty minutes, infectivity was reduced by $90 \%$ at $\mathrm{pH} 5.5$ (Figure 1B). Again, similar losses of infectivity were seen for particles carrying HA. In contrast, no loss of infectivity was seen for pseudovirions carrying the envelope glycoprotein from avian leukosis virus (ALV Env) following incubation for five or thirty minutes at pH 5.0 at all temperatures tested (Figure 1). Finally, consistent with the model in which GP is kinetically trapped in the metastable pre-fusion conformation, no loss of infectivity was observed at any $\mathrm{pH}$ while on ice (Figure 1). These data demonstrate that incubation at pH 5.0 to 5.5 leads to loss of infectivity of GP-bearing pseudovirions at a physiologic temperature.

A

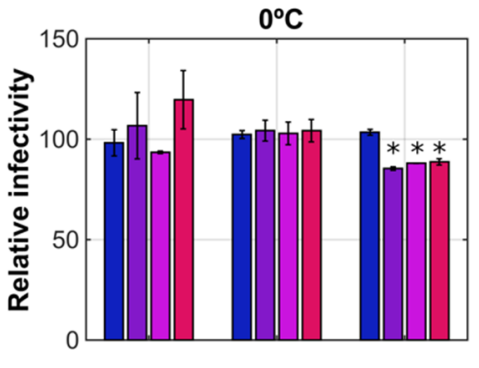

B

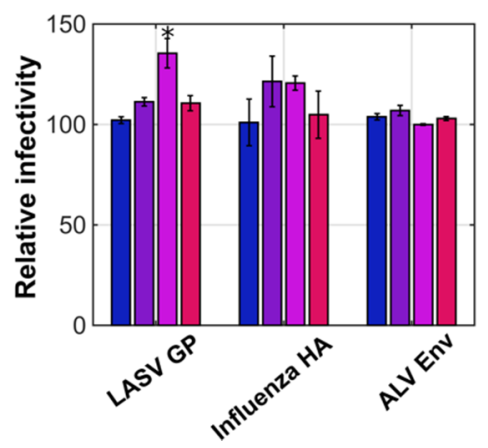

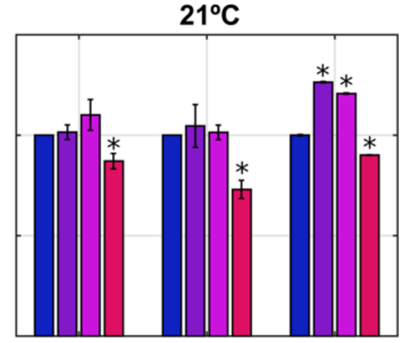
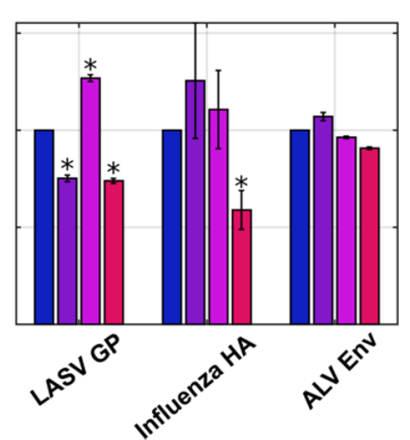

$37^{\circ} \mathrm{C}$
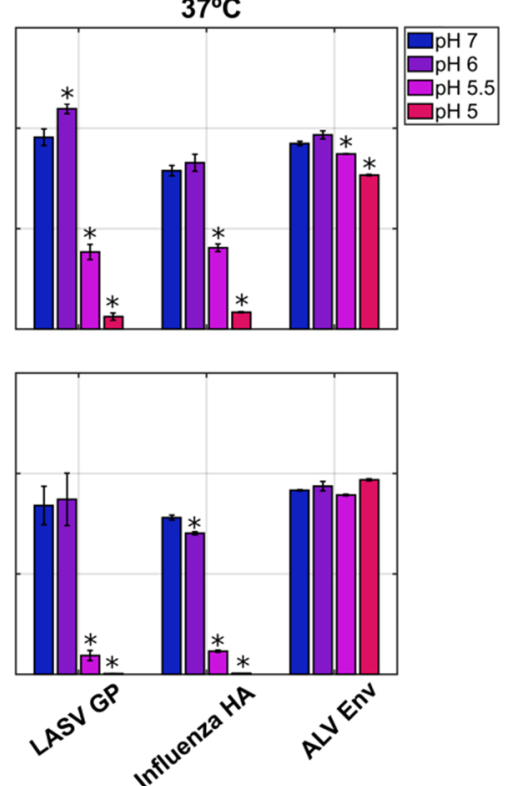

Figure 1. Exposure to low $\mathrm{pH}$ at physiological temperature inactivates LASV GP. VSV $\triangle$ G-GFP-GP viral pseudotypes were treated with either phosphate buffer at $\mathrm{pH} 6.0$ or 7.0, or acetate buffer at $\mathrm{pH} 5.5$ or 5.0 at the indicated temperature for (A) $5 \mathrm{~min}$ or (B) $30 \mathrm{~min}$. Virus was subsequently brought back to neutral $\mathrm{pH}$ and used to infect Vero cells. Infectivity was determined by measuring GFP expression using flow cytometry. Infectivity was normalized to the percent of GFP-positive cells at the $21{ }^{\circ} \mathrm{C}$ $\mathrm{pH} 7.0$ condition for each virus. Assays were performed in biological triplicate with new viral preps, and each biological replicate was performed in technical triplicate. Data are presented as the mean of three biological replicates and error bars represent the standard error of the mean. An asterisk indicates a statistically significant difference as compared to the case of $\mathrm{pH} 7(p<0.05)$ calculated using a one-way ANOVA. The lack of an asterisk indicates no statistically significant difference as compared to $\mathrm{pH} 7$.

\subsection{Acidic $p H$ Triggers Loss of Recognition by Neutralizing Antibodies}

To further test whether exposure to acidic $\mathrm{pH}$ at a physiological temperature triggers a conformational change in LASV GP, we evaluated the recognition by neutralizing antibodies $12.1 \mathrm{~F}$, 37.2D, and 37.7H using an enzyme-linked immunosorbent assay (ELISA). These antibodies recognize epitopes that bridge both GP1 and GP2, making them highly specific for the pre-fusion conformation of GP [13,32]. Virions were incubated at $\mathrm{pH}$ values ranging from 5.0 to 7.0 on ice or at $37^{\circ} \mathrm{C}$ for thirty minutes. The $\mathrm{pH}$ was then neutralized while maintaining temperature, and virions were subsequently 
bound to an ELISA plate at room temperature. We then probed for the presence of the pre-fusion conformation of GP with neutralizing antibodies 12.1F, 37.2D, and 37.7H. Irrespective of temperature, exposure to $\mathrm{pH} 6$ or 7 led to no loss of antibody binding (Figure 2). At pH 5.5, moderate loss of binding by only $37.7 \mathrm{H}$ was seen after a $37^{\circ} \mathrm{C}$ incubation, whereas full binding was maintained after incubation on ice. Incubation at $\mathrm{pH} 5.0$ at $37^{\circ} \mathrm{C}$ abrogated binding by approximately $50 \%$ in the case of $12.1 \mathrm{~F}$, by more than $60 \%$ in the case of $37.2 \mathrm{D}$, and by $75 \%$ in the case of $37.7 \mathrm{H}$, whereas no loss of binding of any antibody was seen after incubation on ice. In contrast, no loss of binding after incubation at either temperature across the range of $\mathrm{pH}$ values was seen for the non-neutralizing antibodies $26.5 \mathrm{E}$ and 24.6C (Figure 2) [32]. Taken together, the infectivity and antibody binding data suggest that GP undergoes a $\mathrm{pH}$-induced transition out of the pre-fusion conformation, concurrent with a loss of function. Here again, only when incubations were performed at a physiological temperature did the putative transition occur at a $\mathrm{pH}$ consistent with the late endosome.

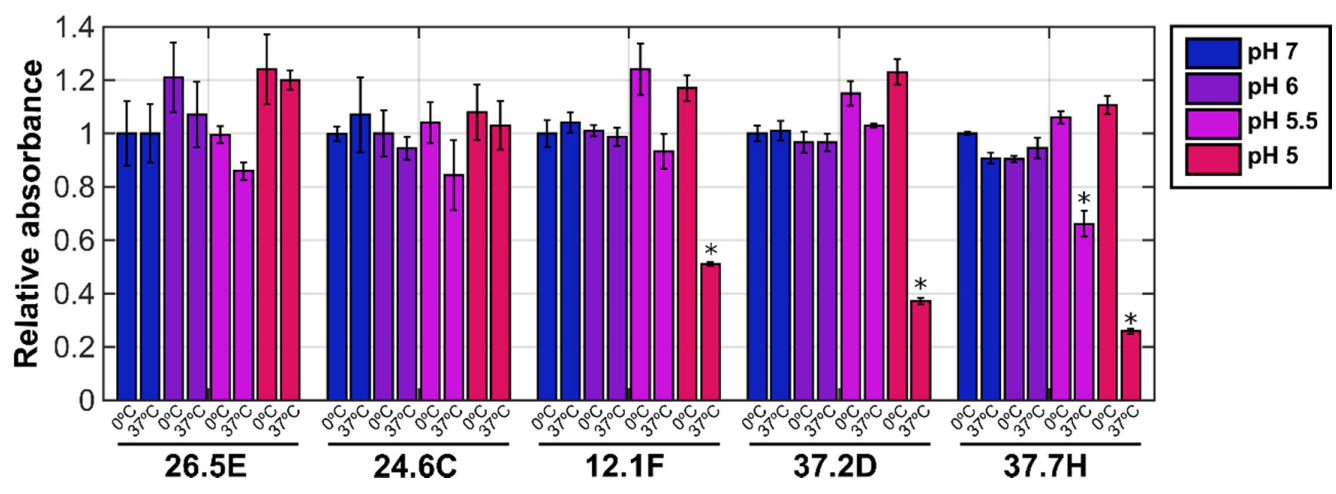

Figure 2. The binding of neutralizing and non-neutralizing antibodies to LASV GP after exposure to acidic $\mathrm{pH}$ at physiological temperature. VSV $\triangle \mathrm{G}-\mathrm{GFP}-\mathrm{GP}$ viral pseudotypes were treated with either phosphate buffer at $\mathrm{pH} 6.0$ or 7.0 , or acetate buffer at $\mathrm{pH} 5.5$ or 5.0 at the indicated temperature for $30 \mathrm{~min}$. Virus was subsequently brought back to neutral $\mathrm{pH}$ and assayed for binding by non-neutralizing $(26.5 \mathrm{E}, 24.6 \mathrm{C})$ and neutralizing antibodies $(12.1 \mathrm{~F}, 37.2 \mathrm{D}, 37.7 \mathrm{H})$ by ELISA [32]. Data are presented as the mean of three biological replicates and error bars represent standard error of the mean. An asterisk indicates a statistically significant difference as compared to the case of incubation at $0{ }^{\circ} \mathrm{C}$ and $\mathrm{pH}$ $7(p<0.05$.) calculated using a one-way ANOVA. The lack of an asterisk indicates no statistically significant difference.

\subsection{Acidic $p H$ Triggers Lipid Mixing Mediated by LASV GP}

Our observations thus far support a hypothesis in which acidic $\mathrm{pH}$ is sufficient to promote the transition of GP from the pre-fusion to the post-fusion conformation. We sought to test this hypothesis by directly visualizing the pH-induced triggering of GP-mediated membrane fusion. To this end, we developed a single-particle lipid mixing assay adapted from those used in studies of influenza [33,37,38], West Nile virus [39], feline coronavirus [40], and VSV [41]. We formed a planar lipid bilayer supported within a quartz microfluidic cell. Contained within the bilayer was lipid-soluble 6-carboxyfluorescein (CF), a pH-sensitive dye used as a fluorescent $\mathrm{pH}$ indicator. VSV-GP pseudovirions were generated as described above, but with the additional inclusion of HA with a mutated furin cleavage site (HA0). This HA0 mutant is incapable of mediating fusion but enabled the attachment of the virions to the lipid bilayer by way of binding sialic acid, which was included in the bilayer in the form of the GD1a ganglioside. This approach allowed us to consider the role of $\mathrm{pH}$ in GP-mediated lipid mixing independently from the engagement of a receptor in the target membrane. The viral membrane was labeled with the lipophilic fluorophore DiD at a concentration sufficient to partially quench fluorescence. Based on the previous applications of this approach, we anticipated that the hemifusion of the viral membrane and the planar bilayer would allow the diffusion of the DiD into the bilayer, which would give rise to a sharp increase in fluorescence as the DiD dequenches. 
The $\mathrm{DiD}$ fluorescence then decays as the dye continues to diffuse into the planar bilayer. We bound VSV-HA0-GP virions to the supported lipid bilayer in the microfluidic channel at $\mathrm{pH} 7.0$ and imaged the surface using prism-based total internal reflection fluorescence (TIRF) microscopy (Figure 3A). Puncta of $\mathrm{DiD}$ fluorescence indicated the presence of virions attached to the surface. Pre-warmed buffers at various $\mathrm{pH}$ values were then introduced into the flow cell with a syringe pump with the continuous monitoring of fluorescence.

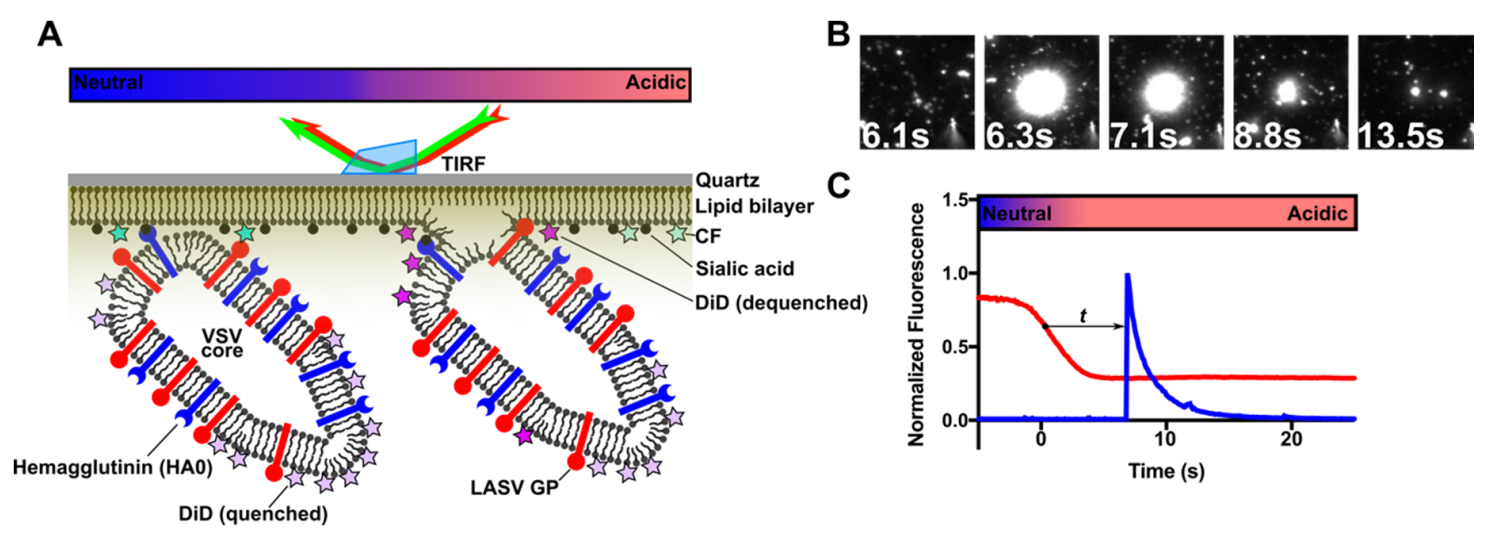

Figure 3. LASV GP mediates lipid mixing between fluorescently labeled virions and a supported lipid bilayer. (A) Cartoon of pseudovirions formed with a VSV core, inactive HA0, and LASV GP, hemifusing to a planar lipid bilayer supported by a quartz microscope slide within a microfluidic chamber. Pseudovirions were flowed onto the bilayer and allowed to attach at neutral $\mathrm{pH}$ by way of the HA0-sialic acid interaction. DiD fluorescence dequenching arose due to single pseudovirions hemifusing to the supported bilayer upon the introduction of acidic $\mathrm{pH}$. CF was included in the supported bilayer in order to provide a fluorescence indicator of $\mathrm{pH}$. Fluorescence was detected using the evanescent field generated from the simultaneous total internal reflection of 642-nm and 532-nm lasers on a custom-built prism-based TIRF microscope. Movies were recorded at a $100 \mathrm{~ms}$ exposure time $(10 \mathrm{~Hz})$ for a minimum of 1400 frames. (B) Frames from a single DiD dequenching event at $\mathrm{pH} 5$ acquired at the indicated time points, where Time $=0$ was defined as a $50 \%$ decrease in CF fluorescence. (C) Representative fluorescence traces (CF, red; DiD, blue) are from a single dequenching event. The drop in CF fluorescence arises from the acidification of the buffer in the channel. The subsequent spike in $\mathrm{DiD}$ fluorescence arises from dequenching as DiD diffuses into the planar bilayer. The interval $(t)$ between the $50 \%$ decrease in CF fluorescence and DiD dequenching was extracted for kinetic analysis.

The introduction of $\mathrm{pH} 4.0$ buffer pre-warmed to $37^{\circ} \mathrm{C}$ induced a precipitous loss of $\mathrm{CF}$ fluorescence, indicating the successful delivery of low $\mathrm{pH}$ buffer to the flow cell. Following the acidification of the chamber, the DiD puncta exhibited the characteristic sharp increase and trailing loss of fluorescence indicative of successful lipid mixing (Figure 3B, Movie S1). The integration of the fluorescence intensity of each punctum allowed for the generation of $\mathrm{CF}$ and DiD fluorescence traces (Figure 3C). Pseudovirions that had been incubated at acidic $\mathrm{pH}$ at $37^{\circ} \mathrm{C}$ prior to binding to the planar bilayer displayed no evidence of lipid mixing upon the introduction of acidic buffer to the flow cell. Similarly, virions lacking GP and possessing only HA0 bound the surface but also did not display any evidence of lipid mixing, indicating that the observed lipid mixing was mediated by GP (Movie S2).

To determine the kinetics of lipid mixing, we extracted the time interval between the drop in CF fluorescence and the dequenching of DiD fluorescence. These intervals were compiled into histograms and fit to a gamma distribution, which allowed the determination of the rate constant, the number of rate-determining steps leading to lipid mixing, and the average time to lipid mixing (Figure 4A, Section Materials and Methods) [33,37]. This fitting procedure indicated that at $\mathrm{pH} 4.0$, there existed approximately $4.2 \pm 0.1$ rate-determining steps leading to lipid mixing, with a rate constant of $0.31 \pm 0.01 \mathrm{~s}^{-1}$, which yielded an average time to lipid mixing of $13.5 \pm 0.5 \mathrm{~s}$. Increasing 
the $\mathrm{pH}$ to 4.5 and 5.0 slowed the rate constant to $0.25 \pm 0.01$ and $0.19 \pm 0.02$, respectively, which led to corresponding increases in the time to lipid mixing (Figure 4B,C, Movies S3,S4). However, across all $\mathrm{pH}$ values considered, the number of rate-determining steps between acidification and lipid mixing was consistently approximately 4 (Figure 4B). No lipid mixing was seen above pH 5.0 on the timescale of our movies. Below pH 4.0, lipid mixing occurred too rapidly to accurately evaluate in this assay. In summary, the timing of lipid mixing occurred in a pH-dependent manner, with the kinetics decreasing with increasing $\mathrm{pH}$. The number of rate-determining steps was not sensitive to $\mathrm{pH}$.
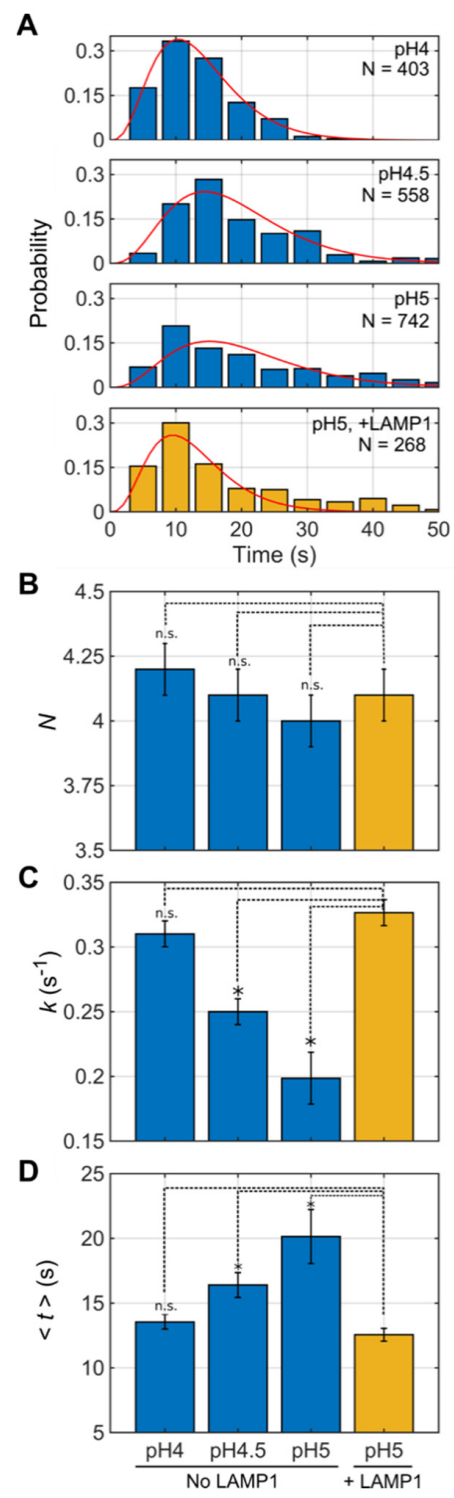

Figure 4. LAMP1 increases the kinetics of GP-mediated lipid mixing. For each lipid mixing event, the interval of time between the 50\% decrease in CF fluorescence and DiD dequenching was compiled into a histogram and fit to a gamma distribution by a least-squares algorithm. (A) The histogram for each $\mathrm{pH}$ considered, in the absence or presence of LAMP1, is shown with the gamma distribution fit overlaid in red. $N$ indicates the number of individual lipid mixing events. (B) For each experiment, the number of rate-determining steps leading from the acidification of the flow cell to dequenching was determined through the gamma distribution fitting of each histogram in A. (C) The rate constants (k) determined from the gamma distribution fitting of each histogram in A. (D) The mean time to dequenching, defined as $N / k$ for a gamma distribution. Error bars reflect the $95 \%$ confidence intervals determined in the fitting. An asterisk indicates a statistically significant difference as compared to the case of LAMP1 at pH $5(p<0.05$.) calculated using a one-way ANOVA. 


\subsection{LAMP1 Increases the Kinetics of Lipid Mixing}

We next asked whether the attachment of the virion to the target membrane by way of GP-LAMP1 interactions would affect the kinetics of lipid mixing. To this end, the single-virion lipid mixing experiment was repeated using VSV-GP pseudovirions lacking HA0, and with a soluble form of LAMP1 immobilized on the planar bilayer by way of a polyhistidine ( $6 \times$ His) tag bound to a Ni-NTA lipid. Virions were bound to the supported bilayer at pH 5.5. As in the absence of LAMP1, pH 5.5 was not sufficiently acidic to trigger lipid mixing. However, rapid lipid mixing was observed at pH 5.0 in the presence of LAMP1 (Movie S5). Kinetic analysis indicated a rate constant and average time to lipid mixing consistent with that observed at $\mathrm{pH} 4.0$ in the absence of LAMP1 (Figure 4). The number of rate-determining steps $(4.1 \pm 0.1)$ remained similar to that seen in the absence of LAMP1 (Figure 4B). Thus, the interaction of GP with LAMP1 increased the kinetics of lipid mixing to an extent equivalent to reducing the $\mathrm{pH}$ by approximately one unit. Here again, pseudovirions that had been pre-incubated at an acidic $\mathrm{pH}$ at $37^{\circ} \mathrm{C}$ prior to binding to the planar bilayer in the presence of LAMP1 displayed no sign of lipid mixing after the introduction of acidic buffer to the flow cell.

\section{Discussion}

Here, we demonstrate that acidic $\mathrm{pH}$ alone is sufficient to induce the conversion of LASV GP to a conformation that is unable to support entry into cells and unable to bind neutralizing antibodies that are specific to the pre-fusion conformation of GP. Importantly, only at a physiological temperature is $\mathrm{pH}$ 5.0, which approximates the $\mathrm{pH}$ of the most acidic endosomes [42], sufficiently acidic to promote this transition. No loss of infectivity or loss of antibody binding was seen when virions were incubated at room temperature or on ice at $\mathrm{pH}$ 5.0. This temperature-dependent transition to an irreversible conformation is consistent with a kinetically trapped pre-fusion conformation of GP. This implies that a large activation energy limits spontaneous transition out of the pre-fusion conformation into a conformation with much lower free energy. Elevated temperature increases the probability of spontaneously crossing this high activation energy, while the protonation of key residues in GP by the acidic environment likely reduces the activation energy. Thus, reduced temperatures, which lower the probability of crossing the activation energy, require more acidic $\mathrm{pH}$ to trigger conformational changes in GP [43]. This underscores the importance of maintaining a physiological temperature when characterizing the activity of glycoproteins such as LASV GP.

By attaching virions to a target membrane by orthogonal means-through HA0-mediated binding to sialic acid-we have further provided direct evidence that acidic $\mathrm{pH}$ is sufficient to trigger LASV GP-mediated lipid mixing in the absence of interaction with either $\alpha$ DG or LAMP1. Thus, no receptor-binding event is strictly necessary to trigger GP-mediated lipid mixing. These data provide support for the previous observation that the disruption of the $\alpha \mathrm{DG}$ binding site through mutagenesis only minimally affects cell-cell fusion [11]. These data also support previous studies indicating that LAMP1 is not necessary for GP-mediated cell-cell fusion [21], nor is it strictly required for entry into cells [20].

Our kinetic analysis of single-virion lipid mixing events indicates that the time from $\mathrm{pH}$ drop to lipid mixing decreases at more acidic $\mathrm{pH}$. This provides direct evidence for the $\mathrm{pH}$-induced reduction in the activation energy that kinetically traps GP in the pre-fusion conformation. Interestingly, although the rate of lipid mixing increased with decreasing $\mathrm{pH}$, the number of rate-determining steps remained approximately constant across the $\mathrm{pH}$ range tested. This indicates that the mechanism of GP-mediated lipid mixing is consistent across a range of $\mathrm{pH}$ values. This may be an indirect observation of intermediate conformations in GP, which may resemble those postulated for other class I viral fusogens, such as influenza HA and Ebola GP $[16,30,43,44]$. A cryo-electron tomography study of GP documented a pH-induced transition of GP to a conformation distinct from the pre- and post-fusion conformations [14], which may reflect an intermediate conformation achieved during fusion. Alternatively, the observation of multiple rate-determining steps leading to lipid mixing may 
be an indication that multiple GP trimers need to engage the target membrane in order to promote lipid mixing.

Finally, we have directly shown that the attachment of virions to the target membrane by way of binding LAMP1 increases the kinetics of lipid mixing. When LAMP1 is present, lipid mixing occurs at $\mathrm{pH} 5.0$ at a rate approximately equal to the rate observed in the absence of LAMP1 at $\mathrm{pH} 4.0$. Thus, while LAMP1 is not necessary for GP to promote lipid mixing, LAMP1 increases the $\mathrm{pH}$ at which GP-mediated lipid mixing occurs, thus facilitating LASV entry under less harsh conditions, as previously suggested [20]. This indicates that LAMP1 and acidic $\mathrm{pH}$ synergistically reduce the activation energy that limits GP conformational changes that lead to fusion. This observation is entirely consistent with previous studies demonstrating that LAMP1 increased the $\mathrm{pH}$ at which GP could mediate cell-cell fusion [21], and that virions enter by way of less acidic endosomes in cells expressing LAMP1 as compared to cells lacking LAMP1 [20]. Taken together, the present and previous studies provide a consistent understanding for the roles that endosomal $\mathrm{pH}$ and LAMP1 play in LASV entry. Future studies will be aimed at probing the mechanism by which acidic $\mathrm{pH}$ and LAMP1 enable GP conformational changes. This will likely require a combination of structure determination and biophysical interrogations equipped to visualize GP conformational changes that lead to fusion.

Supplementary Materials: The following are available online at http://www.mdpi.com/1999-4915/12/7/716/s1, Movie S1. Representative movie of single-virion lipid mixing events that occurred upon exposure to $\mathrm{pH} 4$. The left channel is a visualization of $\mathrm{CF}$ fluorescence used to mark the drop in $\mathrm{pH}$. The right channel is a visualization of the DiD fluorescence, which was used to visualize lipid mixing. Movies were recorded at $100 \mathrm{~ms}$ for a minimum of 1400 frames; Movie S2. Representative movie of the single-virion lipid mixing experiment with VSV pseudotypes bearing only HA0. The movie is presented as for Movie S1; Movie S3. Representative movie of single-virion lipid mixing events that occurred upon exposure to $\mathrm{pH}$ 4.5. The movie is presented as for Movie S1; Movie S4. Representative movie of single-virion lipid mixing events that occurred upon exposure to $\mathrm{pH}$ 5. The movie is presented as for Movie S1; Movie S5. Representative movie of single-virion lipid mixing events that occurred upon exposure to $\mathrm{pH} 5$ in the presence of LAMP1. The movie is presented as for Movie S1.

Author Contributions: U.B. and J.B.M. designed the research and analyzed the data. U.B. and R.G. performed all experiments. All authors wrote and edited the manuscript. All authors have read and agreed to the published version of the manuscript.

Funding: This work was supported by NIH grant DP2AI124384 (to J.B.M.).

Acknowledgments: We wish to thank Melinda Brindley, Peter Kwong, John Coffin, and Juha Huiskonen for kindly providing plasmids for the expression of LASV GP, IAV HA, ALV Env, and LAMP1, respectively. We are also grateful to James Robinson for providing LASV antibodies.

Conflicts of Interest: The authors declare no conflict of interest.

\section{References}

1. Nigeria Centre for Disease Control. Lassa Fever Situation Report; NCDC: Abuja, Nigeria, 2020.

2. Okogbenin, S.; Okoeguale, J.; Akpede, G.; Colubri, A.; Barnes, K.G.; Mehta, S.; Eifediyi, R.; Okogbo, F.; Eigbefoh, J.; Momoh, M.; et al. Retrospective cohort study of Lassa fever in pregnancy, Southern Nigeria. Emerg. Infect. Dis. 2019, 25, 1494-1500. [CrossRef] [PubMed]

3. Mateer, E.J.; Huang, C.; Shehu, N.Y.; Paessler, S. Lassa fever-induced sensorineural hearing loss: A neglected public health and social burden. PLoS Neglect. Trop. D 2018, 12, e0006187. [CrossRef]

4. Hastie, K.M.; Saphire, E.O. Lassa virus glycoprotein: Stopping a moving target. Curr. Opin. Virol. 2018, 31, 52-58. [CrossRef] [PubMed]

5. Lenz, O.; ter Meulen, J.; Klenk, H.D.; Seidah, N.G.; Garten, W. The Lassa virus glycoprotein precursor GP-C is proteolytically processed by subtilase SKI-1/S1P. Proc. Natl. Acad. Sci. USA 2001, 98, 12701-12705. [CrossRef]

6. Burri, D.J.; Pasquato, A.; da Palma, J.R.; Igonet, S.; Oldstone, M.B.A.; Kunz, S. The role of proteolytic processing and the stable signal peptide in expression of the Old World arenavirus envelope glycoprotein ectodomain. Virology 2013, 436, 127-133. [CrossRef]

7. Israeli, H.; Cohen-Dvashi, H.; Shulman, A.; Shimon, A.; Diskin, R. Mapping of the Lassa virus LAMP1 binding site reveals unique determinants not shared by other old world arenaviruses. PLoS Pathog. 2017, 13, e1006337. [CrossRef] [PubMed] 
8. Cao, W.; Henry, M.D.; Borrow, P.; Yamada, H.; Elder, J.H.; Ravkov, E.V.; Nichol, S.T.; Compans, R.W.; Campbell, K.P.; Oldstone, M.B.A. Identification of -dystroglycan as a receptor for lymphocytic choriomeningitis virus and Lassa fever virus. Science 1998, 282, 2079-2081. [CrossRef]

9. Oppliger, J.; Torriani, G.; Herrador, A.; Kunz, S. Lassa virus cell entry via dystroglycan involves an unusual pathway of macropinocytosis. J. Virol. 2016, 90, 6412-6429. [CrossRef]

10. Jae, L.T.; Brummelkamp, T.R. Emerging intracellular receptors for hemorrhagic fever viruses. Trends Microbiol. 2015, 23, 392-400. [CrossRef]

11. Acciani, M.; Alston, J.T.; Zhao, G.; Reynolds, H.; Ali, A.M.; Xu, B.; Brindley, M.A. Mutational analysis of Lassa virus glycoprotein highlights regions required for alpha-dystroglycan utilization. J. Virol. 2017, 91. [CrossRef]

12. Cohen-Dvashi, H.; Cohen, N.; Israeli, H.; Diskin, R. Molecular mechanism for LAMP1 recognition by Lassa virus. J. Virol. 2015, 89, 7584-7592. [CrossRef] [PubMed]

13. Hastie, K.M.; Zandonatti, M.A.; Kleinfelter, L.M.; Heinrich, M.L.; Rowland, M.M.; Chandran, K.; Branco, L.M.; Robinson, J.E.; Garry, R.F.; Saphire, E.O. Structural basis for antibody-mediated neutralization of Lassa virus. Science 2017, 356, 923-928. [CrossRef]

14. Li, S.; Sun, Z.; Pryce, R.; Parsy, M.-L.; Fehling, S.K.; Schlie, K.; Siebert, C.A.; Garten, W.; Bowden, T.A.; Strecker, T.; et al. Acidic $\mathrm{pH}$-induced conformations and LAMP1 binding of the Lassa virus glycoprotein spike. PLoS Pathog. 2016, 12, e1005418. [CrossRef] [PubMed]

15. Baker, D.; Agard, D.A. Influenza hemagglutinin: Kinetic control of protein function. Structure 1994, 2, 907-910. [CrossRef]

16. Harrison, S.C. Viral membrane fusion. Virology 2015, 479, 498-507. [CrossRef] [PubMed]

17. Igonet, S.; Vaney, M.-C.; Vonrhein, C.; Vonhrein, C.; Bricogne, G.; Stura, E.A.; Hengartner, H.; Eschli, B.; Rey, F.A. X-ray structure of the arenavirus glycoprotein GP2 in its postfusion hairpin conformation. Proc. Natl. Acad. Sci. USA 2011, 108, 19967-19972. [CrossRef]

18. Eschli, B.; Quirin, K.; Wepf, A.; Weber, J.; Zinkernagel, R.; Hengartner, H. Identification of an N-terminal trimeric coiled-coil core within arenavirus glycoprotein 2 permits assignment to class I viral fusion proteins. J. Virol. 2006, 80, 5897-5907. [CrossRef]

19. Shulman, A.; Katz, M.; Cohen-Dvashi, H.; Greenblatt, H.M.; Levy, Y.; Diskin, R. Variations in core packing of GP2 from old world mammarenaviruses in their post-fusion conformations affect membrane-fusion efficiencies. J. Mol. Biol. 2019. [CrossRef]

20. Hulseberg, C.E.; Fénéant, L.; Szymańska, K.M.; White, J.M. Lamp1 increases the efficiency of Lassa virus Infection by promoting fusion in less acidic endosomal compartments. Mbio 2018, 9, 75. [CrossRef]

21. Cohen-Dvashi, H.; Israeli, H.; Shani, O.; Katz, A.; Diskin, R. Role of LAMP1 binding and pH sensing by the spike Complex of Lassa virus. J. Virol. 2016, 90, 10329-10338. [CrossRef]

22. Thomas, C.J.; Shankar, S.; Casquilho-Gray, H.E.; York, J.; Sprang, S.R.; Nunberg, J.H. Biochemical reconstitution of hemorrhagic-fever arenavirus envelope glycoprotein-mediated membrane fusion. PLOS ONE 2012, 7. [CrossRef] [PubMed]

23. Borrow, P.; Oldstone, M.B.A. Mechanism of lymphocytic choriomeningitis virus entry into cells. Virology 1994, 198, 1-9. [CrossRef] [PubMed]

24. Glushakova, S.E.; Lukashevich, I.S. Early events in arenavirus replication are sensitive to lysosomotropic compounds. Arch. Virol. 1989, 104, 157-161. [CrossRef] [PubMed]

25. Rojek, J.M.; Sanchez, A.B.; Nguyen, N.T.; de la Torre, J.-C.; Kunz, S. Different mechanisms of cell entry by human-pathogenic Old World and New World arenaviruses. J. Virol. 2008, 82, 7677-7687. [CrossRef] [PubMed]

26. Rojek, J.M.; Kunz, S. Cell entry by human pathogenic arenaviruses. Cell Microbiol. 2008, 10, 828-835. [CrossRef] [PubMed]

27. Cosset, F.-L.; Marianneau, P.; Verney, G.; Gallais, F.; Tordo, N.; Pécheur, E.-I.; ter Meulen, J.; Deubel, V.; Bartosch, B. Characterization of Lassa virus cell entry and neutralization with Lassa virus pseudoparticles. J. Virol. 2009, 83, 3228-3237. [CrossRef]

28. Klewitz, C.; Klenk, H.-D.; ter Meulen, J. Amino acids from both N-terminal hydrophobic regions of the Lassa virus envelope glycoprotein GP-2 are critical for $\mathrm{pH}$-dependent membrane fusion and infectivity. J. Gen. Virol. 2007, 88, 2320-2328. [CrossRef] 
29. Simone, C.D.; Zandonatti, M.A.; Buchmeier, M.J. Acidic $\mathrm{pH}$ triggers LCMV membrane fusion activity and conformational change in the glycoprotein spike. Virology 1994, 198, 455-465. [CrossRef]

30. Das, D.K.; Govindan, R.; Nikić-Spiegel, I.; Krammer, F.; Lemke, E.A.; Munro, J.B. Direct visualization of the conformational dynamics of single influenza hemagglutinin trimers. Cell 2018, 174, 926-937.e12. [CrossRef]

31. Whitt, M.A. Generation of VSV pseudotypes using recombinant $\Delta \mathrm{G}-\mathrm{VSV}$ for studies on virus entry, identification of entry inhibitors, and immune responses to vaccines. J. Virol. Methods 2010, 169, 365-374. [CrossRef] [PubMed]

32. Robinson, J.E.; Hastie, K.M.; Cross, R.W.; Yenni, R.E.; Elliott, D.H.; Rouelle, J.A.; Kannadka, C.B.; Smira, A.A.; Garry, C.E.; Bradley, B.T.; et al. Most neutralizing human monoclonal antibodies target novel epitopes requiring both Lassa virus glycoprotein subunits. Nat. Commun. 2016, 7, 11544. [CrossRef] [PubMed]

33. Floyd, D.L.; Ragains, J.R.; Skehel, J.J.; Harrison, S.C.; van Oijen, A.M. Single-particle kinetics of influenza virus membrane fusion. Proc. Natl. Acad. Sci. USA 2008, 105, 15382-15387. [CrossRef] [PubMed]

34. Scholtissek, C. Stability of infectious influenza A viruses to treatment at low $\mathrm{pH}$ and heating. Arch. Virol. 1985, 85, 1-11. [CrossRef] [PubMed]

35. Marzi, A.; Feldmann, F.; Geisbert, T.W.; Feldmann, H.; Safronetz, D. Vesicular stomatitis virus-based vaccines against Lassa and Ebola viruses. Emerg. Infect. Dis. 2015, 21, 305-307. [CrossRef]

36. Skehel, J.J.; Wiley, D.C. Receptor binding and membrane fusion in virus entry: The influenza hemagglutinin. Annu. Rev. Biochem. 2000, 69, 531-569. [CrossRef]

37. Ivanovic, T.; Choi, J.L.; Whelan, S.P.; van Oijen, A.M.; Harrison, S.C. Influenza-virus membrane fusion by cooperative fold-back of stochastically induced hemagglutinin intermediates. Elife 2013, 2, e00333. [CrossRef]

38. Costello, D.A.; Lee, D.W.; Drewes, J.; Vasquez, K.A.; Kisler, K.; Wiesner, U.; Pollack, L.; Whittaker, G.R.; Daniel, S. Influenza virus-membrane fusion triggered by proton uncaging for single particle studies of fusion kinetics. Anal. Chem. 2012, 84, 8480-8489. [CrossRef]

39. Chao, L.H.; Klein, D.E.; Schmidt, A.G.; Pena, J.M.; Harrison, S.C. Sequential conformational rearrangements in flavivirus membrane fusion. Elife 2014, 3. [CrossRef]

40. Costello, D.A.; Millet, J.K.; Hsia, C.-Y.; Whittaker, G.R.; Daniel, S. Single particle assay of coronavirus membrane fusion with proteinaceous receptor-embedded supported bilayers. Biomaterials 2013, 34, 7895-7904. [CrossRef] [PubMed]

41. Kim, I.S.; Jenni, S.; Stanifer, M.L.; Roth, E.; Whelan, S.P.J.; van Oijen, A.M.; Harrison, S.C. Mechanism of membrane fusion induced by vesicular stomatitis virus G protein. Proc. Natl. Acad. Sci. USA 2016, 114, E28-E36. [CrossRef]

42. Huotari, J.; Helenius, A. Endosome maturation. EMBO J. 2011, 30, 3481-3500. [CrossRef] [PubMed]

43. Carr, C.M.; Chaudhry, C.; Kim, P.S. Influenza hemagglutinin is spring-loaded by a metastable native conformation. Proc. Natl. Acad. Sci. USA 1997, 94, 14306-14313. [CrossRef] [PubMed]

44. Das, D.K.; Bulow, U.; Diehl, W.E.; Durham, N.D.; Senjobe, F.; Chandran, K.; Luban, J.; Munro, J.B. Conformational changes in the Ebola virus membrane fusion machine induced by $\mathrm{pH}, \mathrm{Ca}^{2+}$, and receptor binding. PLoS Biol. 2020, 18, e3000626. [CrossRef] [PubMed]

(C) 2020 by the authors. Licensee MDPI, Basel, Switzerland. This article is an open access article distributed under the terms and conditions of the Creative Commons Attribution (CC BY) license (http://creativecommons.org/licenses/by/4.0/). 